\title{
RETROPERITONEAL HEMANGIOMA: A VERY RARE CASE PRESENTATION
}

\section{Aftab S Shaikh}

Amarjeet E Tandur

Yugal S Pathrabe*

Ajay H Bhandarwar

Tanvi M Shah

Nilofar A Shaikh
Associate Professor, Department of General Surgery Grant Government Medical College \& Sir JJ Group of Hospitals, Mumbai, India

Assistant Professor, Department of General Surgery Grant Government Medical College \& Sir JJ Group of Hospitals, Mumbai, India

Resident, Department of General Surgery Grant Government Medical

College \& Sir JJ Group of Hospitals, Mumbai, India

${ }^{*}$ Corresponding Author

Professor and Head, Department of General Surgery Grant Government Medical College \& Sir JJ Group of Hospitals, Mumbai, India

Senior Resident, Tata Memorial Hospital, Mumbai, India

Consultant Radiologist, Department of Radiology, Mittal Imaging Centre, Mumbai, India

ABSTRACT Retroperitoneal tumour which can be benign or malignant is a rare entity (of which vascular neoplasms are extremely rare) with late clinical presentation, most often diagnosed in advanced stage of the disease. The complex anatomical location in the retroperitoneum, its inaccessibility and close relation to important structures in the retroperitoneal space pose a greater challenge in the diagnosis and the management of the disease. Here we are presenting a case of retroperitoneal tumour which presented with lump in abdomen since 10 years. Biopsy of the lump was suggestive of benign lesion. Complete surgical excision of the tumour was done and on final histopathology was suggestive of benign vascular neoplasm consistent with venous/cavernous hemangioma. Regular follow-up is uneventful.

KEYWORDS : Retroperitoneum, retroperitoneal tumour, hemangioma, cavernous hemangioma, venous hemangioma.

\section{Introduction}

The first case of retroperitoneal hemangioma was reported in 1929. Retroperitoneum is the $2^{\text {nd }}$ most common site of origin of malignant mesenchymal tumours after the lower extremity. Primary retroperitoneal tumours account for 0.07 to $0.6 \%$ of all tumours of which $60-80 \%$ are malignant. Sarcoma comprises one-third of retroperitoneal tumours(Strauss et al., 2011). Benign retroperitoneal tumours are very rare among which primary retroperitoneal hemangioma are extremely rare, however when present cavernous hemangioma are more common whereas venous hemangioma is unusual(Igarashi \& Hanazaki, 1998). Retroperitoneal tumours are best evaluated with cross sectional imaging and pre-operative histology(Strauss et al., 2011), but preoperative diagnosis of a retroperitoneal hemangioma based on imaging is extremely rare. Hemangioma essentially have no risk of malignant transformation(Godar et al., 2013). However, large hemangiomas have potential risk of rupture and massive haemorrhage(Peng et al., 2018)(Forbes, 2005), such as in our case. Surgical excision is the treatment of choice for retroperitoneal tumour(Igarashi \& Hanazaki, 1998)(MARTÍN et al., 2004). We present a case of retroperitoneal lump in a 42 years old male patient who underwent excision of tumour and diagnosed to be hemangioma on histopathology.

\section{Case report}

A 42 years old male patient presented with swelling in the right iliac fossa since 10 years. The swelling was initially small and non-progressive in size but increased in size over the last one and half years, hence the patient took medical consultation. Patient also complained of dull aching type of pain over the region of swelling over the last one and half year. No other significant history or symptom was present. On local examination an ill-defined, firm, non-ballottable mass, that does not move with respiration, of size $8 \times 10 \times 15 \mathrm{cms}$ was present in the right iliac fossa. All laboratory parameters were within normal limits.

Ultrasonography revealed large heterogenous lesion in right iliac fossa with internal foci of calcification and vascularity. Contrast enhanced computed tomography showed well defined lobulated heterogeneously enhancing soft tissue density lesion of size $8.7 \times 10.7 \times 13.3 \mathrm{cms}$ (APxTRxCC) with hypodense component and few calcific foci and marked internal vascularity within it, seen in right iliac fossa. It was producing mass effect in the form of displacement of small and large bowel loops superiorly and to the opposite side. It was displacing the right iliac vessels, mildly compressing the right common iliac vessels and also displacing the right ureter but there was no evidence of obstructive uropathy. The structure from which the tumour originated could not be made (figure 1).

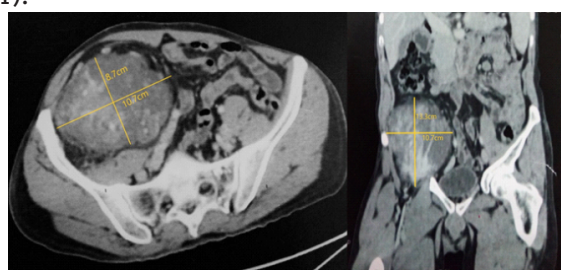

Figure 1-Axial and Coronal Computed Tomography image; tumour mass causing displacement of the bowel loops and adjacent structures upwards and to opposite side.

Ultrasound guided biopsy was done which revealed loose fibrocollagenous tissue with thin and thick walled blood vessels, suggestive of benign etiology.

Contrast enhanced 18F-FDG whole body PET CT scan done revealed Non-FDG avid right iliac fossa mass and metabolically active disease was not present elsewhere in the body. 
Patient was posted for excision of mass. Midline incision was taken. Cattle-Braasch manoeuvre was done to expose the tumour and structures in the retroperitoneum. The tumour was firmly attached to the adjacent structures. Sharp dissection was performed to separate the tumour from blood vessels, ureter and the other important structures. Ligation and cauterization were done to achieve haemostasis whenever required. Mass measuring $12 \times 7 \times 8 \mathrm{cms}$ was excised with a blood loss of around 150-200ml.

The mass was firm and well circumscribed with tiny areas of microcystic change with largest cyst measuring $0.7 \mathrm{~cm}$ in diameter (figure 2). Microscopic examination showed a lowgrade vascular neoplasm composed of variable sized vascular channels with interspersed areas of hyalinisation and fibrosis. There were many large thick-walled dilated blood vessels along with dilated and anastomosing small capillary sized vascular channel. And there were thin walled dilated blood vessels and the lining epithelium of these vascular channel was single layered. No areas of necrosis or haemorrhage was seen on gross or microscopic examination (figure 3). Immunohistochemistry for CD31 and CD34 highlights the endothelial lining of these anastomosing vascular channels. Smooth muscle actin and Desmin highlights the muscle layer of vascular spaces. Histopathological diagnosis of Hemangioma was made. Patient's postoperative period was uneventful and the patient was discharged on 11 th post-operative day.

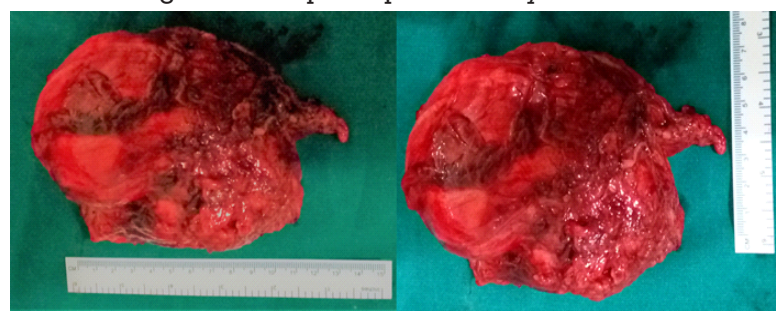

Figure 2-Excised retroperitoneal mass specimen

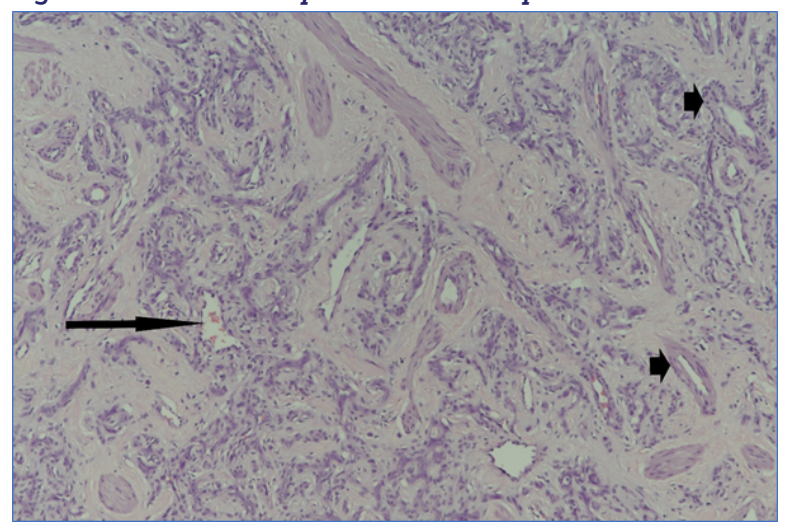

Figure 3- Thin walled large cystically dilated vessels with intravascular thrombosis and lined by single layer of endothelium (large arrow), dilated thick walled vessels (small arrow)

\section{DISCUSSION}

Retroperitoneal space extends from diaphragm superiorly to pelvic brim inferiorly and is situated between posterior parietal peritoneum anteriorly and transversalis fascia posteriorly. It is broadly divided into anterior and posterior pararenal space, perirenal space and the great vessel space. Anterior pararenal space lies between posterior parietal peritoneum and anterior renal fascia of Gerota. Posterior pararenal space lies between posterior renal fascia of Zuckerkandl and transversalis fascia. Perirenal space lies between anterior renal fascia and posterior renal fascia. Anterior renal fascia of Gerota and posterior renal fascia of
Zuckerkandl fuse laterally to form Lateroconal fascia.(Burkill \& Healy, 2000)(Meyers et al., 1972)

Retroperitoneal tumour is an oppressive surgical diagnosis because of its rarity, complex anatomical location, late clinical presentation, difficulty in the diagnosis and recurrence after the excision. Retroperitoneal benign tumours include schwanomma, neurofibroma, paraganglioma, fibromatosis, lipoma, vascular hemangiomas. Malignant tumours include liposarcoma, leiomyosarcoma, lymphoma, malignant fibrous histocytosis, desmoid tumours, exragonadal germ cell tumours and metastatic retroperitoneal lymphadenopathy. Lump in abdomen is the most common symptom, the other complaints with which patients present are anorexia, weight loss, fever, jaundice, haematuria, burning micturition'(Patel, 2020). It is often asymptomatic till the advanced stages and the symptoms that occur late in the course of the disease are due compression of the structures in the retroperitoneum. In our case patient presented with pain in abdomen with displacement of the adjacent structures (but no compression such as ureter causing hydronephrosis or hydroureter)

Retroperitoneal Hemangioma is very rare tumour. There is no obvious gender predisposition for retroperitoneal hemangioma and cases have been reported in both children and adults (Mossanen et al., 2015). Hemangioma is histopathologically classified into capillary, cavernous, arteriovenous and venous types. Retroperitoneal hemangiomas are almost always of cavernous type whereas venous type are extremely rare(Igarashi \& Hanazaki, 1998). Most hemangioamas are present at birth, while some regress spontaneously others grow rapidly during puberty or pregnancy or following trauma and this rapid growth is suggested to be the result of thrombosis and obstruction of outflow of blood(Powis \& Rushton, 1972) which may also have happened in our case.

Preoperative diagnosis of a retroperitoneal hemangioma is unusual and is very difficult. Ultrasonography(US) imaging is usually the first imaging investigation in a case of retroperitoneal tumour. US appearance and echogenicity of hemangioma can be variable, from hyperechoic to anechoic, typically with posterior acoustic enhancement(Godar et al., 2013).Contrast enhanced computed tomography(CECT) is the imaging of choice for a retroperitoneal tumour(Strauss et al., 2011 )(Wee-Stekly \& Mueller, 2014).CECT provides the tumour size, position, extent and adjacent organ involvement. Magnetic resonance imaging (MRI)is playing an increasing role in the evaluation of retroperitoneal soft-tissue masses due to its excellent tissue characterization and spatial resolution. In general, the dynamic enhancement patterns in MRI reflect the vascularity of masses, differentiating benign from malignant retroperitoneal tumours(Elsayes et al., 2007). The tissue-specific multiplanar capability of high-resolution MRI allows better tumour localization and internal characterization, thereby serving as a road map for surgical planning (Shanbhogue et al., 2012). The few reported cases of hemangioma in whom MRI was performed revealed a low signal intensity on the Tl-weighted image and high signal intensity on the T2-weighted image(Igarashi \& Hanazaki, 1998)(Matsuoka et al., 2018) and have been suggested to represent the haemorrhage and hyalinization of tissue (Mossanen et al., 2015). Malignant vascular tumours such as angiosarcoma, epithelioid hemangioendothelioma, and Kaposi sarcoma resemble hemangiomas ,but these tumours often have low signal central regions on T2-weighted images or multiple lesions distinguishing typical features of hemangioma(Worawattanakul et al., 1997)(Buetow et al., 1994).The imaging findings of most retroperitoneal soft tissue tumours are nonspecific and a definitive diagnosis most often is established only by histopathologic evaluation. Imaging analysis of the tumour size, extent, components, growth pattern and vascularity can assist in identification of a more specific differential diagnosis(Osman et al., 2013). 
On gross pathological examination in most reported cases, the tumour revealed multiloculated cysts filled with blood and is suggested to be the result of repeated haemorrhages (Hanaoka et al., 2013) (Mundinger et al., 2009). However, in our case no gross areas of haemorrhage and necrosis were seen and only small areas of cystic change were noted, might be in the initial stage. Microscopically, a cavernous hemangioma consist of thin walled large cystically dilated vessels with intravascular thrombosis and are lined by single layer of endothelium(He et al., 2012). However, in our case many large dilated thick-walled vessels were also seen which is more likely to be found in a venous hemangioma rather than a cavernous hemangioma. Also in contrast to cavernous hemangiomas, smooth muscle cells in venous hemangiomas are detected histologically in the vessel walls(Igarashi \& Hanazaki, 1998). But a definitive distinction between a cavernous or venous hemangioma could not be made. Surprisingly in our case the hemangioma did not complicate into bleeding on a preoperative biopsy.

On immunohistochemistry, positive stain for vascular endothelial markers CD31 and CD34 supports the diagnosis of hemangioma (Mundinger et al., 2009) (Godar et al., 2013) (He et al., 2012)(Forbes, 2005).

Hemangiomas are essentially non-malignant but large hemangioma as in our case have high risk of rupture and bleeding. Though rare, cases of retroperitoneal haemorrhage secondary to ruptured retroperitoneal hemangioma have been reported (Peng et al., 2018) (Forbes, 2005). As majority of retroperitoneal tumours are malignant requiring histopathological diagnosis, surgical excision is the treatment of choice for retroperitoneal tumours (Igarashi \& Hanazaki, 1998) (Godar et al., 2013). In our case the tumour produced mass effect in the form of displacement of adjacent organs and intraoperatively no adjacent organs were invaded and hence excision could be done without resection of adjacent organs. However cases have been reported for hemangioma causing invasion and destruction of the adjacent organs such as duodenum and pancreas and therefore needed combining the resection of the surrounding organs(Hanaoka et al., 2013).

\section{Conclusion}

Retroperitoneal hemangioma is very rare tumour. Despite the advances in the imaging studies preoperative diagnosis of a retroperitoneal hemangioma remains a concern and a diagnosis can be made only on postoperative histopathology. A prompt and immediate intervention should be done in a case of large retroperitoneal tumour to avoid complications such as compression and haemorrhage.

\section{REFERENCES}

1. Buetow, P. C., Buck, J. L., Ros, P. R., \& Goodman, Z. D. (1994). Malignant vascular tumors of the liver: radiologic-pathologic correlation. Radiographics : A Review Publication of the Radiological Society of North America, Inc, 14(1). https://doi.org/10.1148/radiographics.14.1.8128048

2. Burkill, G. J. C., \& Healy, J. C. (2000). Anatomy of the retroperitoneum. Imaging, 12(1), 10-20. https://doi.org/10.1259/img.12.1.120010

3. Elsayes, K. M., Staveteig, P. T., Narra, V. R., Chen, Z. M., Moustafa, Y. L., \& Brown, J. (2007). Retroperitoneal Masses: Magnetic Resonance Imaging Findings with Pathologic Correlation. In Current Problems in Diagnostic Radiology (Vol. 36, Issue 3, pp. 97-106). Mosby. https:// doi.org/ 10.1067/ j. cpradiol. 2006.12.003

4. Forbes, T. L. (2005). Retroperitoneal hemorrhage secondary to a ruptured cavernous hemangioma. Canadian Journal of Surgery. Journal Canadien de Chirurgie, 48(1), 78-79. http://www.ncbi.nlm.nih.gov/pubmed/15757047

5. Godar, M., Yuan, Q., Shakya, R., Xia, Y., \& Zhang, P. (2013). Case Report Mixed Capillary Venous Retroperitoneal Hemangioma. Case Reports in Radiology, 2013. https://doi.org/10.1155/2013/258352

6. Hanaoka, M., Hashimoto, M., Sasaki, K., Matsuda, M., Fujii, T., Ohashi, K., \& Watanabe, G. (2013). Retroperitoneal cavernous hemangioma resected by a pylorus preserving pancreaticoduodenectomy. World Journal of Gastroenterology, 19(28), 4624-4629. https://doi.org/10.3748/wjg.v19.i28.4624

7. He, H., Du, Z., Hao, S., Yao, L., Yang, F., Di, Y., Li, J., Jiang, Y., Jin, C., \& Fu, D. (2012). Adult primary retroperitoneal cavernous hemangioma: a case report. https://doi.org/10.1186/1477-7819-10-261

8. Igarashi, J., \& Hanazaki, K. (1998). Retroperitoneal venous hemangioma. American Journal of Gastroenterology, 93(11), 2292-2293. https:// doi.org/ 10.
$1111 / j .1572-0241.1998 .00642 . x$

9. MARTIN R. N. P. ZARRANZ J. E., FERNANDEZ M. del C. V. REDONDO, C. C. SESMERO, J. A., \& MARTINEZ-SAGARRA, J. (2004). Laparoscopic Resection of Retroperitoneal Venous Hemangioma. Journal of Urology, 171(1), 336-336. https://doi.org/10.1097/01.ju.0000101996.84249.f3

10. Matsuoka, Y., Kato, T., \& Sugimoto, M. (2018). A case of retroperitoneal vascular malformation. Urology Case Reports, 21, 75-77. https:// doi. org/ 10. 1016/j.eucr.2018.09.003

11. Meyers, M. A., Whalen, J. P., Peelle, K., \& Berne, A. S. (1972). Radiologic features of extraperitoneal effusions. An anatomic approach. Radiology, 104(2), 249-257. https://doi.org/10.1148/104.2.249

12. Mossanen, M., Dighe, M., Gore, J., \& Mann, G. (2015). Large retroperitoneal hemangioma encompassing the renal vein. Journal of the Canadian Urological Association, 9(11-12December), E894-E896. https:// doi.org/ 10. 5489/cuaj.3356

13. Mundinger, G. S., Gust, S., Micchelli, S. T., Fishman, E. K., Hruban, R. H., \& Wolfgang, C. L. (2009). Case Report Adult Pancreatic Hemangioma: Case Report and Literature Review. Gastroenterology Research and Practice, 839730. https://doi.org/10.1155/2009/839730

14. Osman, S., Lehnert, B. E., Elojeimy, S., Cruite, I., Mannelli, L., Bhargava, P., \& Moshiri, M. (2013). A Comprehensive Review of the Retroperitoneal Anatomy, Neoplasms, and Pattern of Disease Spread. In Current Problems in Diagnostic Radiology (Vol. 42, Issue 5, pp. 191-208). Mosby. https:// doi.org/10.1067/j.cpradiol.2013.02.001

15. Patel, K. (2020). Role of computed tomography scan in diagnosis of retroperitoneal masses. International Surgery Journal, 7(5), 1461. https:// doi.org/10.18203/2349-2902.isj20201852

16. Peng, X., Luo, W., Zhang, X., \& Zhu, W. (2018). Sudden onset flank pain: A case report of retroperitoneal hemorrhage secondary to a ruptured adrenal hemangioma. Journal of Pain Research, 11, 1421-1424. https:// doi. org/ 10. 2147/JPR.S160661

17. Powis, S. J. A., \& Rushton, D. I. (1972). A case of retroperitoneal haemangioma British Journal of Surgery, 59(1), 74-76. https://doi.org/10.1002/bjs.180059012

18. Shanbhogue, A. K., Fasih, N., Macdonald, D. B, Sheikh, A. M., Menias, C. O \& Prasad, S. R. (2012). Uncommon primary pelvic retroperitoneal masses in adults: A pattern-based imaging approach. Radiographics, 32(3), 795-817. https://doi.org/10.1148/rg.323115020

19. Strauss, D. C., Hayes, A. J., \& Thomas, J. M. (2011). Retroperitoneal tumours: Review of management. Annals of the Royal College of Surgeons of England, 93(4), 275-280. https://doi.org/10.1308/003588411X571944

20. Wee-Stekly, W. W., \& Mueller, M. D. (2014). Retroperitoneal Tumors in the Pelvis: A Diagnostic Challenge in Gynecology. In Frontiers in Surgery (Vol. 1). Frontiers Media S.A. https://doi.org/10.3389/fsurg.2014.00049

21. Worawattanakul, S., Semelka, R. C., Kelekis, N. L. \& Woosley, J. T. (1997) Angiosarcoma of the liver: MR imaging pre- and post-chemotherapy. Magnetic Resonance Imaging, 15(5), 613-617. https://doi.org/10.1016/S0730 725X(96)00393-1 